\title{
American College of Rheumatology Criteria for Improvement in Rheumatoid Arthritis
}

National Cancer Institute

\section{Source}

National Cancer Institute. American College of Rheumatology Criteria for Improvement

in Rheumatoid Arthritis. NCI Thesaurus. Code 197983.

Standard criteria to compare the effectiveness of various arthritis medications or treatments. The American College of Rheumatology criteria consists of a variety of measures such as, the number of tender joints, number of swollen joints, patient's assessment of pain, patient's and physician's global assessments of disease activity, patient's assessment of physical function, and laboratory evaluation of an acute-phase reactant. 